\title{
NIR-Responsive Lysosomotropic Phototrigger: 'AIE + ESIPT' Active Naphthalene Based Single Component Photoresponsive Nanocarrier with Two-Photon Uncaging and Real-Time Monitoring Ability
}

\author{
Biswajit Roy, ${ }^{\mathrm{a}}$ Rakesh Mengji, ${ }^{\mathrm{b}, \mathrm{c}}$ Samrat Roy, ${ }^{\mathrm{d}}$ Bipul Pal, ${ }^{\mathrm{d}}$ Avijit Jana, ${ }^{* b, c}$ N. D. Pradeep Singh ${ }^{* a}$ \\ ${ }^{a}$ Department of Chemistry, Indian Institute of Technology Kharagpur, Kharagpur-721302, India. \\ bepartment of Organic Synthesis and Process Chemistry, CSIR-Indian Institute of Chemical Technology Hyderabad, Uppal \\ Road, Hyderabad 500007, India. \\ cAcademy of Scientific and Innovative Research (AcSIR), Ghaziabad 201002, India. \\ ${ }^{\mathrm{d}}$ Department of Physics, Indian Institute of Science Education and Research Kolkata, Mohanpur, West Bengal 741246, India.
}

KEYWORDS: Lysosome targeting, Drug delivery system, Nanoparticle, Two-photon uncaging, AIE, ESIPT

\begin{abstract}
In recent times, organelle targeted drug delivery systems gained tremendous attention due to the site specific delivery of active drug molecules resulting in enhanced bioefficacy. In this context, the phototriggered drug delivery system (DDS) for releasing an active molecule is superior as it provides spatial and temporal control over the release. So far, near infrared (NIR) light responsive organelle targeted DDS has not yet been developed. Hence, we introduced a two-photon NIR-light responsive lysosome targeted 'AIE + ESIPT' active single component DDS based on naphthalene chromophore. The Two-photon absorption cross-section of our DDS is $142 \mathrm{GM}$ at $850 \mathrm{~nm}$. The DDS was converted into pure organic nanoparticles for biological applications. Our nanoDDS is capable of selective targeting, AIE-luminogenic imaging, and drug release within the lysosome. In vitro studies using cancerous cell lines showed that our single component photoresponsive nanocarrier exhibited enhanced cytotoxicity and real-time monitoring ability of the drug release.
\end{abstract}

\section{INTRODUCTION}

Lysosome was discovered in 1955 by Christian de Duve. ${ }^{1}$ It is a single membranous subcellular organelle and ubiquitous in almost all eukaryotic cells. ${ }^{2}$ Lysosomes play essential roles in digestion, foreign substance scavenging, and autophagy to maintain cellular homeostasis. ${ }^{3}$ The acidic environment $(\mathrm{pH} 4.5-$ 5.5) of the lysosomes helps digest all types of macromolecules. ${ }^{2,3}$ Disruption of lysosomal functions leads to several diseases. Fifty types of monogenic diseases are related to lysosomal dysfunction. Most of them fall in the category of lysosomal storage diseases (LSDs). Other important diseases are Alzheimer's disease, autoimmune diseases, and resistance to autoimmune diseases. ${ }^{4}$ When the lysosomal degradative pathway gets dysregulated, diseases like cancer are known to progress. ${ }^{5}$

Researchers have recently focused on developing lysosome-targeted drug delivery systems (DDSs) mainly because many of the drug molecules need to be specifically localized in the lysosome to exhibit their maximum activity. ${ }^{4}$ Further, several drugs suffer from multidrug resistance (MDR) due to lysosomal autophagy. ${ }^{6}$ Importantly, targeting the lysosome ensures facile intercellular drug release. Because of these reasons, lysosomes become a vital organelle for targeted drug delivery. ${ }^{3}$ Hence, several $\mathrm{pH}$-responsive and enzyme-responsive drug delivery systems were reported, which specifically released the drug within the lysosome due to its acidic and enzyme-enriched environment. ${ }^{7}$ However, these delivery systems lack control over the drug uncaging process. In this context, light triggered DDSs have gained considerable importance in the last few decades because they provide high spatio-temporal control over the drug release. ${ }^{8}$

Recently, lysosome-targeted light responsive DDSs based on coumarin $^{9 \mathrm{a}}$ and BODIPY ${ }^{9 \mathrm{~b}}$ chromophore have been reported. Riezman et al. developed for the first time a lysosome-targeted coumarin-based DDS to uncage sphingosine, a lipid molecule by UV-light illumination. ${ }^{9 a}$ They investigated the localizationdependent metabolism of the lipid molecule and found a distinct metabolic pattern of lysosomal sphingosine. Later, Weinstain et al. reported several organelle-targeted visible light-responsive photocages based on meso-methyl BODIPY backbone. ${ }^{9 \mathrm{~b}}$ They discovered that the predesigned photocages exhibit organellespecific localization and higher efficacy of the active molecule upon release.

To date, near infrared (NIR) light responsive organelle targetable DDS has not been developed. By any means, if we can develop NIR responsive organelle targetable DDS, we can improve the drug efficacy and eliminate the competitive absorptions of light by natural pigments like hemoglobin. As we know, light below $650 \mathrm{~nm}$ is primarily absorbed by hemoglobin. ${ }^{8}$ Hence, we intend to design a two-photon responsive lysosomotropic DDS that can be operated in the NIR region. With twophoton excitation, the active molecule can be released only at the focal point of the laser, which enables three-dimension 
Scheme 1. Working protocol of Lyso-Naph-Cbl.
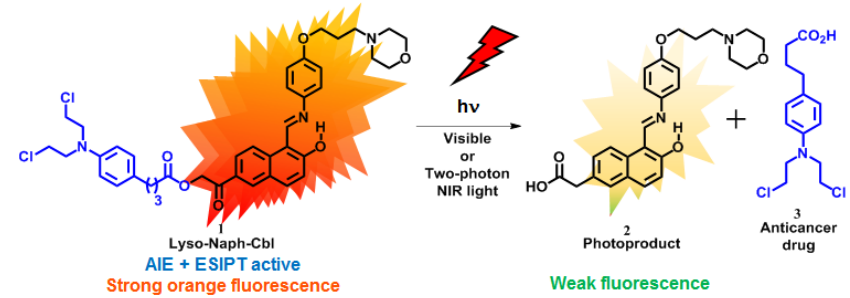

control over uncaging with high spatial and temporal precision. ${ }^{10}$

Herein, we designed a lysosome targeted two-photon responsive 'aggregation-induced emission (AIE) + excited-state intramolecular proton-transfer (ESIPT)' active single chromophoric drug delivery system (Scheme 1), named lysosomotropic-naphthalene-chlorambucil conjugate (Lyso-Naph-Cbl 1). LysoNaph-Cbl consists of a two-photon active 2-hydroxy-6napthacyl backbone ${ }^{11}$ assembled with an imine-sidearm at 1position of naphthalene and caged alkylating agent chlorambucil. Our DDS (Lyso-Naph-Cbl) exhibits the following advantages: (i) due to the presence of an imine linker, the adjacent hydroxyl group takes part in ESIPT process, ${ }^{12}$ and also persuades AIE property, ${ }^{13}$ thereby, providing better visualization (ii) targets lysosome due to installed morpholine moiety which acts as a lysosomotropic anchor ${ }^{3}$ (iii) releases the drug molecule upon single-photon excitation by visible light irradiation as well as upon two-photon excitation with NIR light irradiation and (iv) capable of monitoring drug release in real-time by a sharp decrease in the fluorescence intensity.

\section{RESULTS AND DISCUSSION}

Synthesis of Lyso-Naph-Cbl. We synthesized our DDS starting from commercially available 2-hydroxy-1-naphthaldehyde (4). Friedel-Crafts acylation on 4 with bromoacetyl bromide in the presence of $\mathrm{AlCl}_{3}$ gave compound $\mathbf{5}$. The bromo derivative 5 was then esterified with chlorambucil using $\mathrm{K}_{2} \mathrm{CO}_{3}$ as a base in acetonitrile to furnish compound $\mathbf{6}$. Then to attach the sidearm at 1-position, we separately synthesized the amine compound 7 starting with 4-nitrophenol (7a) following the known procedure ${ }^{14}$ (Scheme 2). Then compounds 6 and 7 were condensed via Dean-Stark distillation in benzene to afford our DDS Lyso-Naph-Cbl 1. All the synthesized compounds were characterized using ${ }^{1} \mathrm{H} \mathrm{NMR}$,

Scheme 2. Synthesis of Lyso-Naph-Cbl.

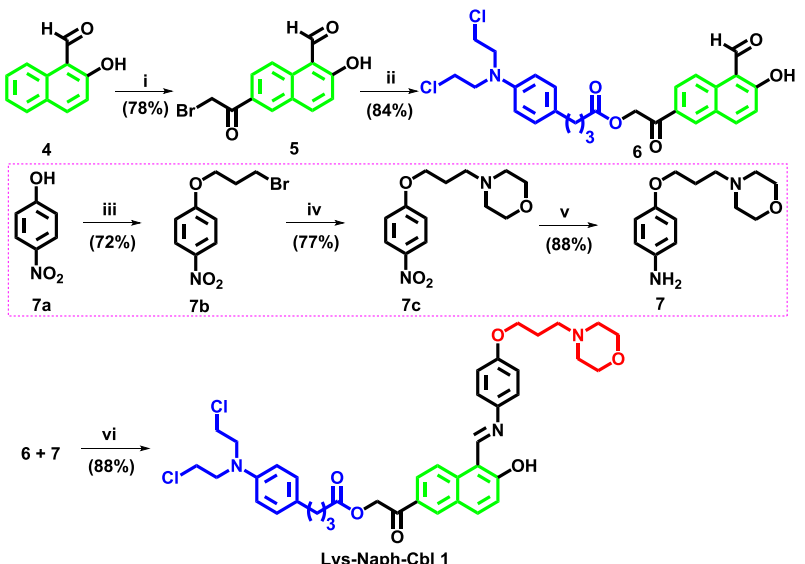

Lys-Naph-Cbl 1

Reaction condition: i) bromoacetyl bromide, $\mathrm{AlCl}_{3}$, dry DCM, $0{ }^{\circ} \mathrm{C}, 5 \mathrm{~h}$; ii) chlorambucil, $\mathrm{K}_{2} \mathrm{CO}_{3}$ acetonitrile, rt, $4 \mathrm{~h}$; (iii) 1,3-dibromopropane, $\mathrm{K}_{2} \mathrm{CO}_{3}$, dry DMSO, $70^{\circ} \mathrm{C}, 4 \mathrm{~h}$; (iv) morpholine, $\mathrm{K}_{2} \mathrm{CO}_{3}$, dry DMSO, $80^{\circ} \mathrm{C}, 5 \mathrm{~h}$; (v) $\mathrm{SnCl}_{2} 2 \mathrm{H}_{2} \mathrm{O}$, EtOAc, reflux, 3h; (vi) Dean-Stark, $4 \mathrm{~h}$.
${ }^{13} \mathrm{C}$ NMR and HRMS (see the Supporting Information, pages S5-S17).

Photophysical properties of Lyso-Naph-Cbl. The photophysical properties of Lyso-Naph-Cbl were investigated in different solvents (Figure 1). The results showed that LysoNaph-Cbl exhibited two absorption maxima due to the ESIPT process $^{12}$. Absorption maxima at $390 \mathrm{~nm}$ and $460 \mathrm{~nm}$ corresponds to the keto-form and enol-form of Lyso-Naph-Cbl (Figure 1a), respectively. However, we noted the absorption maximum corresponding to the enol form to be intense in polar protic solvents. Further, we also noted that keto and enol forms of Lyso-Naph-Cbl showed emission maxima at 480 and 530 $\mathrm{nm}$, respectively (Figure 1b).

To demonstrate the AIE property of our DDS, we recorded the fluorescence intensity of Lyso-Naph-Cbl in acetonitrile-buffer $(\mathrm{pH}$ 7.4) binary mixtures with varying water fractions (Figure 1c-d). Initially, Lyso-Naph-Cbl showed a green fluorescence that, upon increasing the water fraction ( $\mathrm{fw}$ ), got intense (Figure 1d). Further increase of water fraction above 80 to 97 percent, the emission maximum exhibited a large bathochromic shift of around $110 \mathrm{~nm}$ with 6 times enhancement in fluorescence intensity which proved the existence of AIE property. ${ }^{13}$
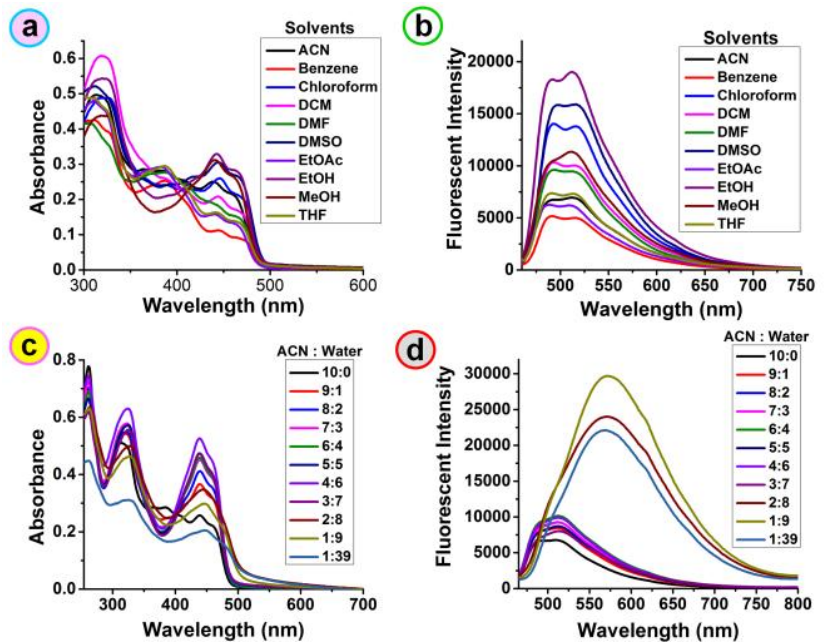

Figure 1. (a) UV-Vis absorbance and (b) fluorescence spectra of Lys-NaphCbl $\left(1 \times 10^{-5} \mathrm{M}\right)$ in different solvents; (c) UV-Vis absorbance and (d) fluorescence spectra of Lyso-Naph-Cbl $\left(1 \times 10^{-5} \mathrm{M}\right)$ in acetonitrile-water binary mixtures with varying proportions.

Stability of Lyso-Naph-Cbl. We checked the stability of LysoNaph-Cbl in a biological medium and two other pHs (5.4 \& 8) at $37^{\circ} \mathrm{C}$ under dark conditions. From Table S1, we observed that no significant amount of drug was released by our DDS after 7 days.

Photouncaging of chlorambucil from Lyso-Naph-Cbl. To check our DDS can release caged chlorambucil upon light irradiation, we investigated its single-photon uncaging ability and quantum yield of the photorelease. A $40 \mathrm{~mL}\left(1 \times 10^{-4} \mathrm{M}\right)$ solution of Lyso-Naph-Cbl in $\left(f_{w}=95 \%\right)$ ACN/PBS buffer ( $\mathrm{pH}$ $=7.4)$ mixture was irradiated with visible light $(\lambda \geq 410 \mathrm{~nm})$ from a $125 \mathrm{~W}$ medium pressure mercury lamp as the light source using a $1(\mathrm{M})$ solution of $\mathrm{NaNO}_{2}$ as the UV-cutoff filter. We monitored the photouncaging process by reverse-phase high-performance liquid chromatography (RP-HPLC) and emission spectroscopy. From the RP-HPLC diagram (Figure $2 \mathrm{a}$ ), we observed that the peak at $t_{R}$ (retention time) $=3.3$ was gradually decreasing, indicating the photo-decomposition of Lyso-Naph-Cbl. On the other hand, we noted 

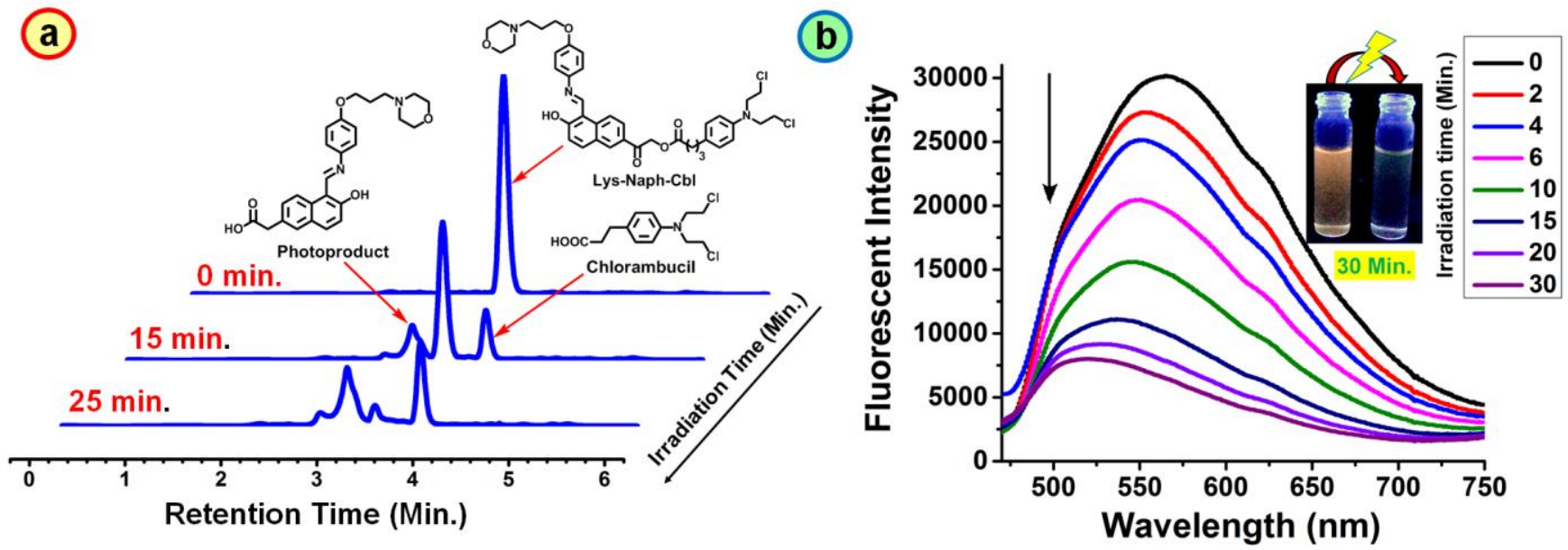

Figure 2. (a) HPLC overlay chromatogram of Lyso-Naph-Cbl at different time intervals of light irradiation ( $\geq 410 \mathrm{~nm}$ ); (b) Change in the fluorescence spectral profile of Lyso-Naph-Cbl with increasing irradiation time.

a continuous increase of two new peaks at $t_{R}=3.0$ and $t_{R}=3.8$, indicating photoproduct formation and uncaged chlorambucil. Figure 2 a shows that $90 \%$ of the drug got released by DDS in 25 minutes of irradiation (Figure S16a). To check that the uncaging process depends only upon the light irradiation, we performed the photouncaging process in the presence and absence of light. Figure S16b showed that photouncaging of chlorambucil is solely dependent on light irradiation. ${ }^{11,15}$ Next, we recorded the emission spectra during the photolysis.

Interestingly, we noted a sharp decrease in the emission maximum during the photouncaging. Initially, our DDS exhibited bright orange fluorescence, and after 25 minutes of irradiation, DDS showed faint fluorescence. The initial peak at $570 \mathrm{~nm}$ of DDS gradually decreased, and a five times decrease in fluorescence intensity was observed (Figure 2b). The above changes might be attributed to the loss of conjugation in the photoproduct (2) due to the Photo-Favorskii rearrangement ${ }^{11}$ (Scheme 1). A similar photouncaging study of Lyso-Naph-Cbl was also carried out at lysosomal $\mathrm{pH}$. We noted a faster uncaging (20 $\mathrm{min}$ ) at lower $\mathrm{pH}=5.4$ (Figure $\mathrm{S} 16 \mathrm{a}$ ), which is a characteristic of Photo-Favorskii rearrangement. ${ }^{8,11}$ The quantum yield of photouncaging $\left(\phi_{\mathrm{u}}\right)$ at different $\mathrm{pHs}$ was calculated by potassium ferrioxalate actinometery ${ }^{16}$ and found to be 0.19 and 0.24 at pHs 7.4 and 5.4, respectively.

Mechanism of photorelease from Lyso-Naph-Cbl. Based on the above experimental findings, literature reports, ${ }^{8,12,17}$ and our recent work on 2-hydroxy-6-napthacyl phototrigger, ${ }^{11}$ we proposed a possible mechanism of photouncaging of Lyso-

Scheme 3. Possible photorelease mechanism of Lyso-Naph-Cbl.

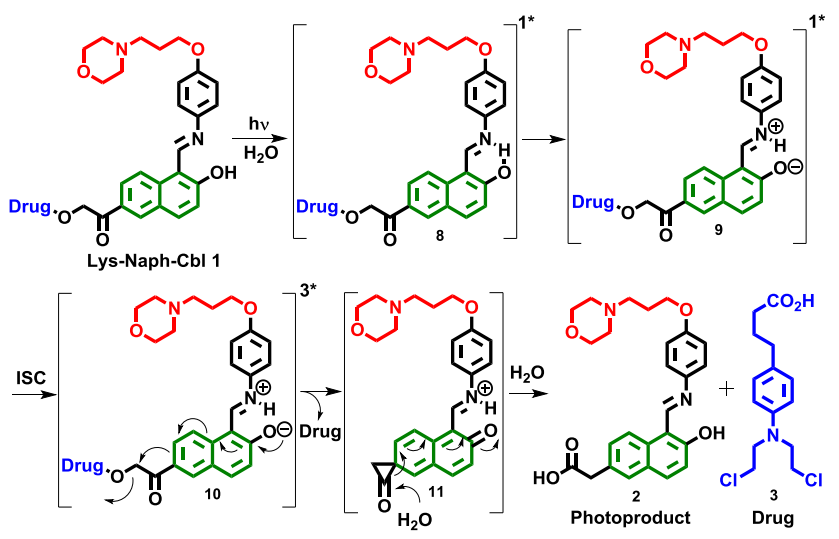

Naph-Cbl (Scheme 3). Upon irradiation, Lyso-Naph-Cbl gets excited to its singlet excited state $\mathbf{8}$ where it undergoes a rapid ESIPT process and generates a zwitterionic form $\mathbf{9}$, which then undergoes efficient intersystem crossing to its triplet excited state 10. A Photo-Favorskii type rearrangement occurs from the triplet state to form a putative intermediate $\mathbf{1 1}$ and subsequent release of chlorambucil. Finally, water reacts with the putative intermediate, resulting in the formation of photoproduct (2). Further, the formation of the photoproduct (2) and released chlorambucil (3) was confirmed by HRMS (Figure S17-S19).

To prove that the reaction proceeds via a triplet state, we carried out the photolysis in the presence of potassium sorbate, a triplet quencher (Figure S20). ${ }^{11,12}$ We found that the uncaging of chlorambucil practically arrested, which confirms that the photolysis proceeds via triplet state.

Measurement of TPA cross-section $\delta_{a}$ of Lyso-Naph-Cbl. Next, we investigated the two-photon absorption ability of Lyso-Naph-Cbl. Using a single beam open aperture (OA) zscan technique, ${ }^{18}$ we performed the nonlinear optical measurement of Lyso-Naph-Cbl. A Ti: Sapphire laser giving 100 fs pulses with $1 \mathrm{~W}$ average power and a focused beam spotsize of $50 \mu \mathrm{m}$ was used for the OA $\mathrm{z}$-scan technique. OA z-scan measurements were carried out on $10^{-4} \mathrm{M}$ solutions of LysoNaph-Cbl in ACN/PBS buffer ( $\mathrm{pH} 7.4$ ) binary mixtures with varying water fractions $\left(f_{\mathrm{W}}\right)$ at different wavelengths, ranging from $700-850 \mathrm{~nm}$ (Figure 3).

The experimental OA curves were then fitted with the transmission equation, including two-photon absorption (TPA). ${ }^{18}$ TPA cross-section $\delta_{\mathrm{a}}$ of Lyso-Naph-Cbl was then calculated $^{19}$ and summarized in Table S2 \& Figure 3f. From Table $\mathrm{S} 2$, we noticed that our DDS has a good TPA cross-section in the NIR region. The highest value of $\delta_{\mathrm{a}}$ for Lyso-Naph-Cbl was obtained at $850 \mathrm{~nm}$, which was $142 \mathrm{GM}\left(1 \mathrm{GM}=10^{-50} \mathrm{~cm}^{4}\right.$ s/photon/molecule). We calculated the two-photon uncaging cross-section $\delta_{\mathrm{u}}$ to understand the efficacy of drug release by two-photon irradiation. The single-photon $\phi_{\mathrm{u}}$ was used to calculate $\delta_{\mathrm{u}}$ by the equation $\delta_{\mathrm{u}}=\phi_{\mathrm{u}} \delta_{\mathrm{a}}{ }^{15}$ The highest $\delta_{\mathrm{u}}$ value for our DDS obtained at $850 \mathrm{~nm}$ was $27 \mathrm{GM}$ and $34 \mathrm{GM}$ at $\mathrm{pH} 7.4$ and 5.4, respectively.

Two-photon uncaging of chlorambucil from Lyso-NaphCbl. The two-photon uncaging of Lyso-Naph-Cbl was demonstrated at two different pHs, 7.4 and 5.4. A $300 \mu \mathrm{L}\left(10^{-4}\right.$ M) solution of Lyso-Naph-Cbl in $f_{w}=95 \%$ ACN/PBS buffer 
a

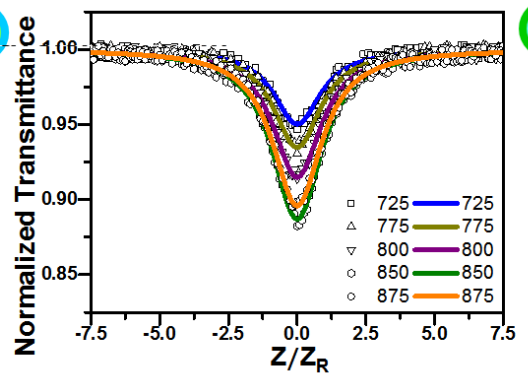

(d)

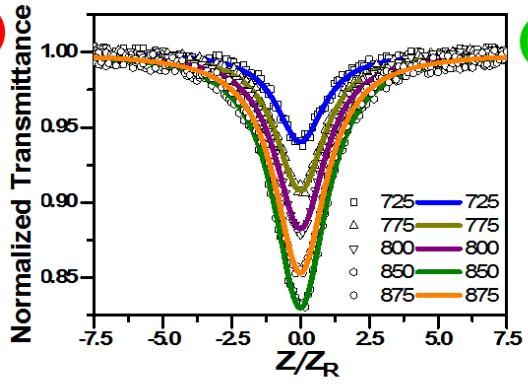

(b)

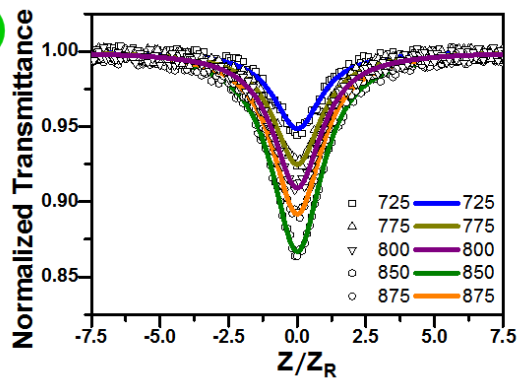

(e)

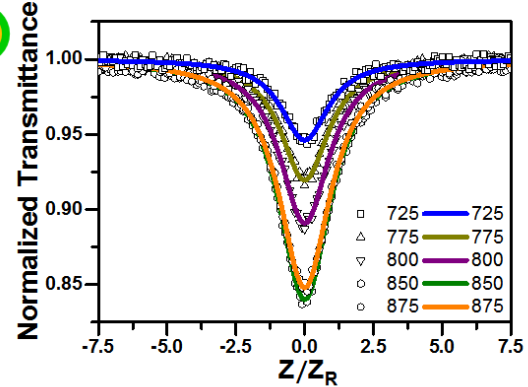

(C)

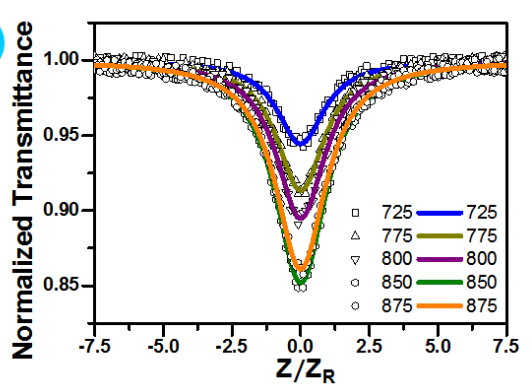

(f)

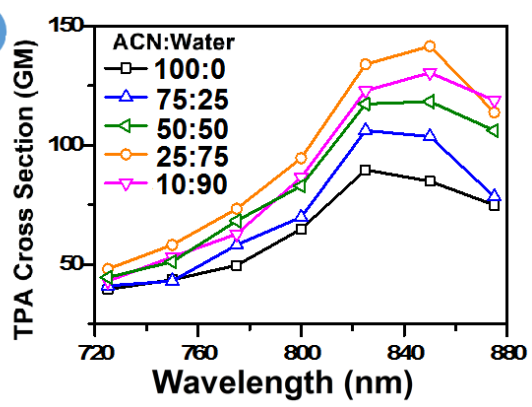

Figure 3. (a-e) OA z-scan curves recorded at different wavelengths using a $10^{-4} \mathrm{M}$ solution of Lyso-Naph-Cbl in ACN:PBS buffer binary mixture with varying water fractions $\left(f_{\mathrm{W}}\right)$; (f) comparison of TPA cross-section of Lyso-Naph-Cbl at different wavelengths and in different $f_{\mathrm{W}}$.

was irradiated with a $100 \mathrm{fs}$ pulsed laser with $50 \mu \mathrm{m}$ focussed beam size and $800 \mathrm{~mW}$ average power at a wavelength of 850 $\mathrm{nm}$. The released chlorambucil was quantified from the HPLC study and found that $24 \%$ and $29 \%$ of the drug got released after $3 \mathrm{~h}$ of irradiation at $\mathrm{pH} 7.4$ and 5.4, respectively.

In vitro applicability of Lyso-Naph-Cbl as nano DDS. The positive outcome of our DDS encouraged us to investigate its targeting, imaging, and drug release capability by in vitro studies using cancer cell lines.

At first, we prepared pure organic nanoparticles (NPs) of LysoNaph-Cbl by reprecipitation technique by following our previously reported procedure for biological application (Figure 4a). ${ }^{20}$ To find the size and shape of Lyso-Naph-Cbl-NPs, we took transmission electron microscopy (TEM). Figures 4a and $4 \mathrm{~b}$ revealed that the Lyso-Naph-Cbl-NPs are almost spherical, with an average size around $\sim 6 \mathrm{~nm}$.
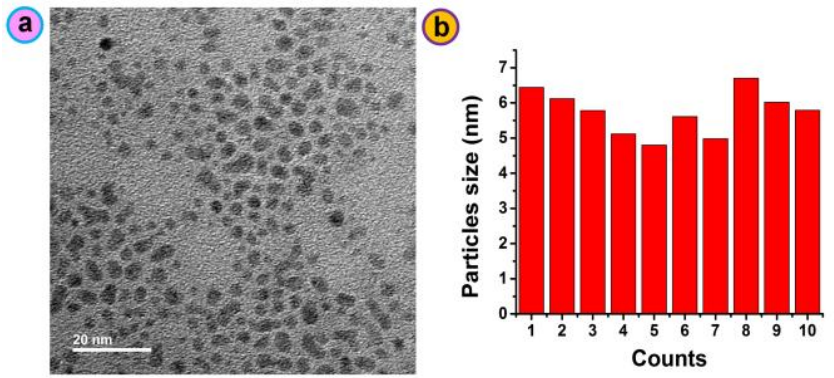

Figure 4: (a) TEM image of DCM-VPA-NPs; (b) Average size of the DCM-VPA-NPs.

Next, we examined the targeting and cell imaging potential of our nano-DDS using cancerous cell line B16F10. To understand its cytoplasmic distribution, Lyso-Naph-Cbl-NPs were counterstained with 4,6-diamidino-2-phenylindole (DAPI), mitotracker green (MTG), and with lysotracker green (LTG), separately. The confocal laser-scanning microscope (CLSM) images (Figure 5) showed that Lyso-Naph-Cbl is mainly colocalized with LTG (Figure 5c) but not with MTG (Figure 5a). (a)
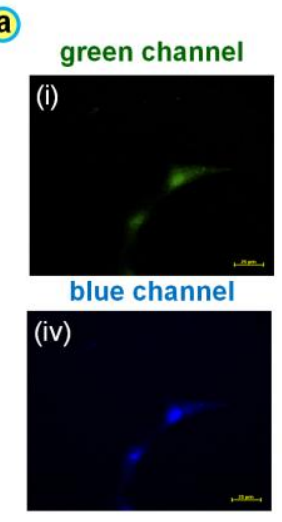

(C)
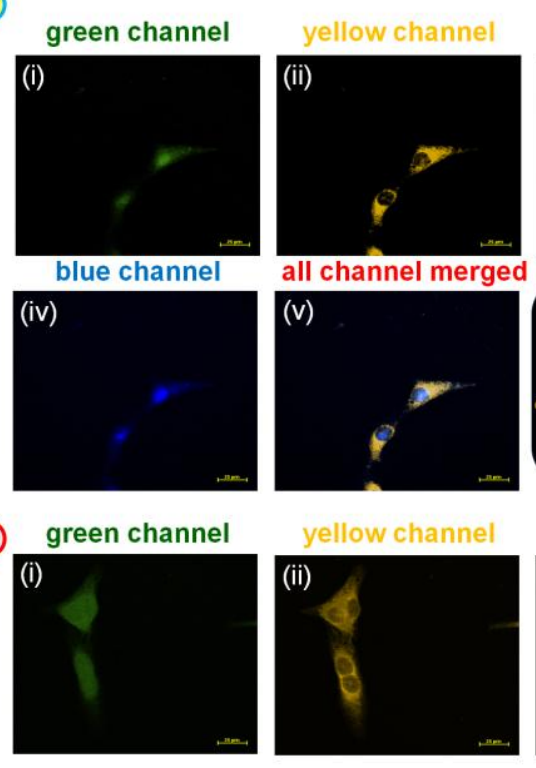

green + yellow channel merged

Figure 5: (a) Live B16F10 cell lines treated with MTG, DAPI and LysoNaph-Cbl-NPs and CLSM images were collected via [a(i)] green channel, [a(ii)] yellow channel, [a(iii)] merged image of green \& yellow channel, [a(iv)] blue channel, [a(v)] merged image of all channels; (b) Structure of Lyso-Naph-Cbl; (c) B16F10 cell lines treated with LTG and Lyso-NaphCbl-NPs and CLSM images were collected via [c(i)] green channel, [c(ii)] yellow channel, [c(iii)] merged image of green \& yellow channel.

After the cellular internalization study of Lyso-Naph-Cbl-NPs, we investigated the in vitro drug uncaging ability of our nanoDDS by light irradiation. B16F10 was incubated with LysoNaph-Cbl-NPs, and the cell nuclei were stained with DAPI. After $1 \mathrm{~h}$ of incubation, we irradiated the cells with visible light $(\lambda$ $\geq 410 \mathrm{~nm}$ ) for 25 minutes. CLSM images (Figure 6b) showed that before irradiation Lyso-Naph-Cbl-NPs exhibited intense fluorescence color (Figure 6b-ii). After irradiation for $25 \mathrm{~min}$, the fluorescence intensity decreased sharply (Figure 6b-v), which validates the photoresponsive drug delivery within the lysosome of the cells. 
(a)

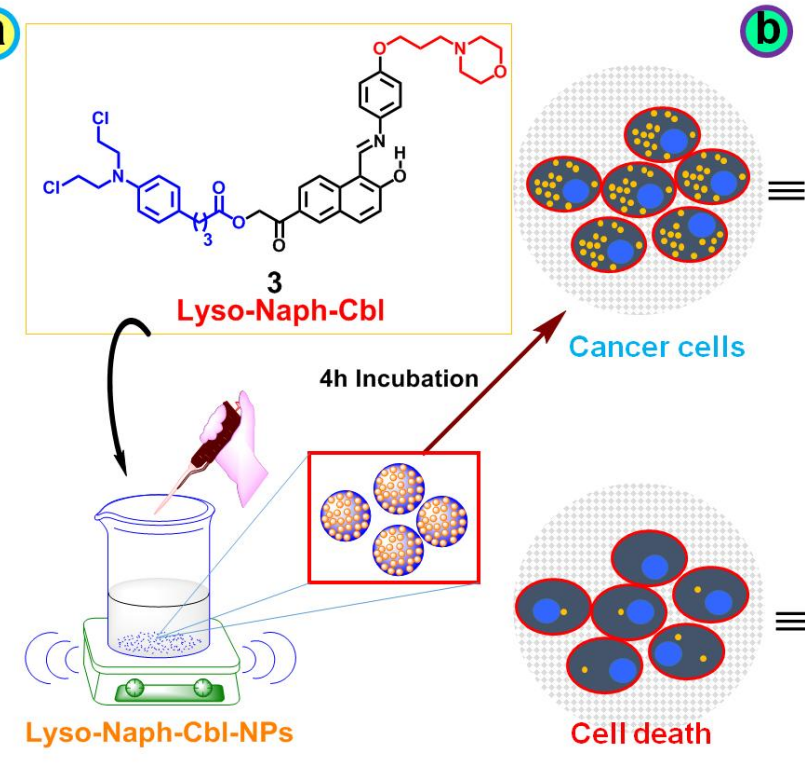

Blue chanell
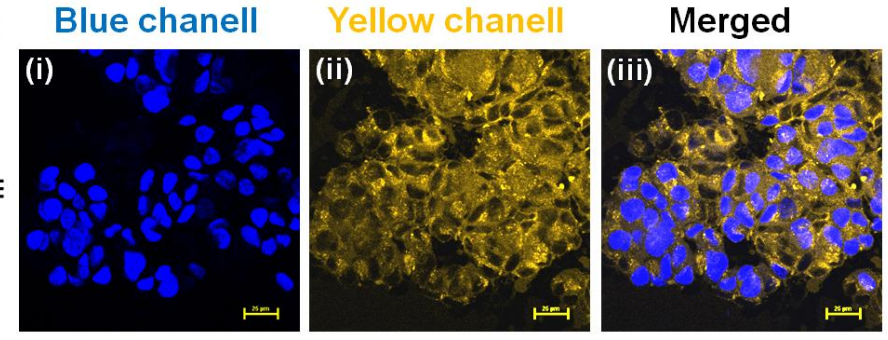

\begin{tabular}{c|c}
$\mathrm{h} v$ \\
$\geq 410 \mathrm{~nm}$
\end{tabular} \mid $\begin{gathered}\text { Irradiation for } \\
25 \mathrm{~min} .\end{gathered}$
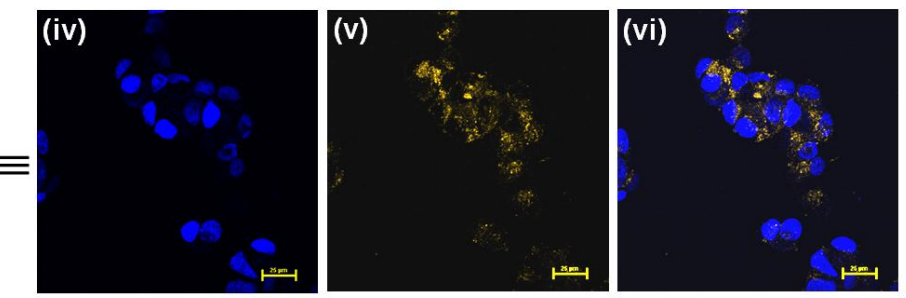

Figure 6: (a) Schematic representation of the preparation of DCM-VPA-NPs; (b) B16F10 cell lines treated with DAPI and Lyso-Naph-Cbl-NPs and CLSM images were collected before irradiation via; [b(i)] blue channel, [b(ii)] yellow channel, [b(iii)] merged image of blue \& yellow channel and after irradiation via; [b(iv)] blue channel, [b(v)] yellow channel, [b(vi)] merged image of blue \& yellow channel.

Then, we carried out the cytotoxicity study with Lyso-NaphCbl-NPs by MTT assay using cancerous cell line B16F10. We found above $80 \%$ cell viability at different concentrations of Lyso-Naph-Cbl (Figure 7a). Next, we irradiated the LysoNaph-Cbl-NPs treated B16F10 cells with visible light and found enhanced cytotoxicity of Lyso-Naph-Cbl-NPs compared to free chlorambucil (Figure 7b). The enhanced cytotoxicity can be explained by the cytosolic drug delivery, validated by MTT data.
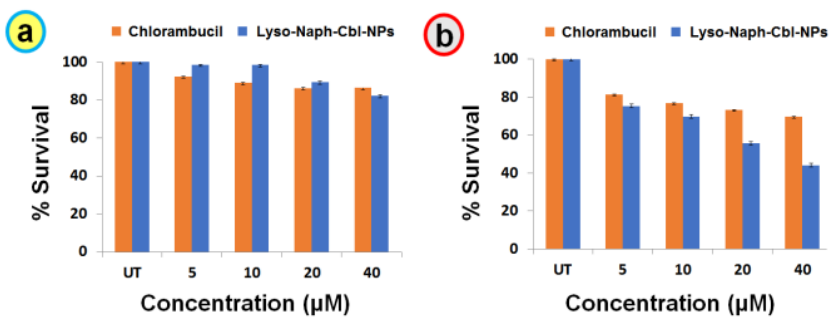

Figure 7: Cell viability assays for Lyso-Naph-Cbl-NPs and free chlorambucil with B16F10 cell line (a) in the dark and (b) after light irradiation for 25 minutes.

In conclusion, for the first time, we developed an organelle targeted two-photon NIR light responsive DDS based on naphthalene chromophore. The DDS was converted into pure organic nanoparticles, targeting the lysosome and releasing the drug molecule inside the cancerous cells upon irradiation. Interestingly, our nano-DDS showed real-time monitoring of the drug release by a sharp decrease in the fluorescence intensity. Finally, the nano-DDS exhibited enhanced cytotoxicity compared to free chlorambucil due to cytosolic drug delivery. Our phototrigger can be utilized to design DDS for targeting other organelles by changing the targeting moiety via simple synthetic modifications.

\section{EXPERIMENTAL SECTION}

Photolysis of Lyso-Naph-Cbl. A $30 \mathrm{~mL}\left(1 \times 10^{-4} \mathrm{M}\right)$ solution of Lyso-Naph-Cbl in acetonitrile/PBS buffer of $\mathrm{pH} 7.4\left(f_{\mathrm{W}}=95\right)$ was taken and degassed prior to exposure to a medium pressure mercury lamp $(125 \mathrm{~W})$ as the source of light $(\lambda \geq 410 \mathrm{~nm})$ using $1(\mathrm{M})$ aqueous solution of $\mathrm{NaNO}_{2} \mathrm{UV}$ cut-off filter.

Two-photon photolysis. The two-photon uncaging of LysoNaph-Cbl was demonstrated with two different solutions of $\mathrm{pHs}$ 7.4 and 5.4. A $300 \mu \mathrm{L}\left(1 \mathrm{X} 10^{-4} \mathrm{M}\right)$ solution of Lyso-Naph-Cbl in $\left(f_{w}=95 \%\right)$ acetonitrile/PBS buffer of $\mathrm{pH} 7.4$ and 5.4 was irradiated with a 100 fs pulsed laser with $50 \mu \mathrm{m}$ focal beam size and $800 \mathrm{~mW}$ average power at a wavelength of $850 \mathrm{~nm}$. A small aliquots $(25 \mu \mathrm{L})$ before and after the photolysis were taken out from the solution for the HPLC study. The released drug was quantified from the HPLC peak area in comparison with an injected authentic sample.

Preparation of nanoparticles of Lyso-Naph-Cbl. LysoNaph-Cbl was dissolved in THF to prepare a $1 \times 10^{-3} \mathrm{M}$ solution. Next, $25 \mu \mathrm{L}$ solution was added dropwise (1 drop per minute) via syringe into $2.5 \mathrm{~mL}$ water in a glass beaker with constant sonication. Sonication was continued for 30 minutes at room temperature. Then the beaker was heated to $37^{\circ} \mathrm{C}$, and nitrogen was purged through the solution for $1 \mathrm{~h}$ to remove the THF to give a $1 \times 10^{-5} \mathrm{M}$ solution of Lyso-Naph-Cbl-NPs.

Cell lines. Cancerous cell lines B16F10 were obtained from the National Centre for Cell Science (NCCS), Pune, India. All the cell lines were cultured in Dulbecco's Modified Eagle's Medium (DMEM) media (Gibco) supplemented with $10 \%$ fetal bovine serum, $1 \%$ non-essential amino acid, $1 \%$ streptomycin, $1 \%$ L-glutamine, and $1 \%$ penicillin. All cells were cultured at $37^{\circ} \mathrm{C}$ in a $\mathrm{CO}_{2}$ incubator (Thermo Fisher Scientific, USA).

Intracellular distribution of Lyso-Naph-Cbl-NPs in cancerous cell line B16F10. Intracellular localization of Lyso-NaphCbl-NPs by cancerous B16F10 cell line was monitored by confocal laser scanning microscopy (CLSM). The cells were cultured following the standard protocols and were incubated with the Lyso-Naph-Cbl-NPs (10 $\mu \mathrm{M}$ in HEPES buffer) under $5 \%$ $\mathrm{CO}_{2}$ at humified conditions at $37{ }^{\circ} \mathrm{C}$ for $4 \mathrm{~h}$. The cell nucleus was stained with 4,6-diamidino-2-phenylindole (DAPI). Cell mitochondria and lysosomes were stained separately with myto-tracker green and lyso-tracker green, respectively. Then 
imaging was done by Nikon confocal microscope (Eclipse TiE) using the respective filter.

Fluorogenic real-time monitoring of drug release study with B16F10 cells. B16F10 cell lines were cultured following the standard protocols and were treated with the Lyso-Naph-CblNPs (10 $\mu \mathrm{M}$ in HEPES buffer), and the cell nuclei were stained with 4,6-diamidino-2-phenylindole (DAPI). Cells were incubated at $37{ }^{\circ} \mathrm{C}$ under $5 \% \mathrm{CO}_{2}$ at humified conditions for $4 \mathrm{~h}$. After that, cells were irradiated with visible light $(\geq 410 \mathrm{~nm}$ ) for $25 \mathrm{~min}$. Fluorescent images were captured before and after the irradiation using confocal microscopy (Nikon Ti Eclipse).

In Vitro cell viability assay. The in vitro cytotoxic studies of Lyso-Naph-Cbl-NPs and the free drugs chlorambucil (Cbl) have been carried out using the MTT (3-(4,5- dimethylthiazole2-yl)-2,5-diphenyltetrazolium bromide) assay. Briefly, cancerous cell line B16F10 was grown in their log phase. Cells were seeded in 96-well plates in Dulbecco's modified Eagle's medium (DMEM) containing $10 \%$ fetal bovine serum (FBS) for 8 $\mathrm{h}$. The cell lines were then incubated with different concentrations $(5-40 \mu \mathrm{M})$ of Lyso-Naph-Cbl-NPs and Cbl separately in HEPES buffer, at $37{ }^{\circ} \mathrm{C}$ in $5 \% \mathrm{CO}_{2}$ for $4 \mathrm{~h}$. Next, we irradiated these cells with visible light for $25 \mathrm{~min}$ and incubated for $72 \mathrm{~h}$. Then cytotoxicity was measured using the MTT assay before and after light irradiation.

\section{ACKNOWLEDGMENT}

We thank DST SERB (Grant No. EMR/2016/005885 and $\mathrm{SERB} / \mathrm{F} / 6429 / 2020-21)$ for financial support. The CSIR-IICT Communication number for this manuscript is IICT/Pubs./2021/196

\section{REFERENCES}

(1) de Duve, C.; Pressman, B. C.; Gianetto, R.; Wattiaux, R.; Appelmans, F. Tissue Fractionation Studies. 6. Intracellular Distribution Patterns of Enzymes in Rat-Liver Tissue. Biochem. J., 1955, 60, 604-617. (2) Zhu, H.; Fan, J.; Du, J.; Peng, X. Fluorescent Probes for Sensing and Imaging within Specific Cellular Organelles. Acc. Chem. Res., 2016, 49, 2115-2126.

(3) Xu, W.; Zeng, Z.; Jiang, J.-H.; Chang, Y.-T.; Yuan, L. Discerning the Chemistry in Individual Organelles with Small-Molecule Fluorescent Probes. Angew. Chem. Int. Ed., 2016, 55, 13658 - 13699.

(4) Sakhrani, N. M.; Padh, H. Organelle targeting: third level of drug targeting. Drug Des. Dev. Ther., 2013, 7, 585-599.

(5) Dielschneider, R. F.; Henson, E. S.; Gibson, S. B. Lysosomes as Oxidative Targets for Cancer Therapy. Oxid. Med. Cell. Longev, 2017, 2017, 1-8.

(6) (a) Hu, F.; Liu, B. Organelle-specific bioprobes based on fluorogens with aggregation-induced emission (AIE) characteristics. Org. Biomol. Chem., 2016, 14, 9931-9944. (b) Li, Y.-J.; Lei, Y.-H.; Yao, N.; Wang, C.-R.; Hu, N.; Ye, W.-C.; Zhang, D.-M.; Chen, Z.-S. Autophagy and multidrug resistance in cancer. Chin. J. Cancer., 2017, 36, 52.

(7) (a) Manchun, S.; Dass, C. R.; Sriamornsak, P. Targeted therapy for cancer using $\mathrm{pH}$-responsive nanocarrier systems. Life Sci., 2012, 90, 381-387. (b) He, X.; Li, J.; An, S.; Jiang, C. pH-sensitive drug-delivery systems for tumor targeting. Ther. Deliv., 2013, 4, 1499-1510. (c) Dong, H.; Pang, L.; Cong, H.; Shen, Y.; Yu, B. Application and design of esterase-responsive nanoparticles for cancer therapy. Drug Deliv., 2019, 26, 416-432. (d) Cao, Z.; Li, W.; Liu, R.; Li, X.; Li, H.; Liu, L.; Chen, Y.; Lv, C.; Liu, Y. pH- and enzyme-triggered drug release as an important process in the design of anti-tumor drug delivery systems. Biomed. Pharmacother., 2019, 118, 109340. (e) de la Rica, R.; Aili, D.; Stevens, M. M. Enzyme-responsive nanoparticles for drug release and diagnostics. Adv. Drug Deliv. Rev., 2012, 64, 967-978.
(8) Klán, P.; Šolomek, T.; Bochet, C. G.; Blanc, A.; Givens, R.; Rubina, M.; Popik, V.; Kostikov, A.; Wirz, J. Photoremovable Protecting Groups in Chemistry and Biology: Reaction Mechanisms and Efficacy. Chem. Rev. 2013, 113, 119-191.

(9) (a) Feng, S.; Harayama, T.; Chang, D.; Hannich, J. T.; Winssinger, N.; Riezman, H. Lysosome-targeted photoactivation reveals local sphingosine metabolism signatures. Chem. Sci., 2019, 10, 2253-2258. (b) Kand, D.; Pizarro, L.; Angel, I.; Avni, A.; Friedmann-Morvinski, D.; Weinstain, R. Organelle-Targeted BODIPY Photocages: VisibleLight-Mediated Subcellular Photorelease. Angew. Chem. Int. Ed. 2019, $58,4659-4663$.

(10) (a) Piant, S.; Bolze, F.; Specht, A. Two-photon uncaging, from neuroscience to materials. Opt. Mater. Express 2016, 6, $1679-1691$. (b) Abe, M.; Chitose, Y.; Jakkampudi, S.; Thuy, P.; Lin, Q.; Van, B.; Yamada, A.; Oyama, R.; Sasaki, M.; Katan, C. Design and Synthesis of Two-Photon Responsive Chromophores for Near-Infrared Light-Induced Uncaging Reactions. Synthesis 2017, 49, 3337-3346.

(11) Roy, B.; Kundu, M.; Singh, A. K.; Singha, T.; Bhattacharya, S.; Datta, P. K.; Mandal, M.; Singh, N. D. P. Stepwise dual stimuli triggered dual drug release by a single naphthalene based two-photon chromophore to reverse MDR for alkylating agents with dual surveillance in uncaging steps. Chem. Commun. 2019, 55, 13140-13143.

(12) Barman, S.; Mukhopadhyay, S. K.; Biswas, S.; Nandi, S.; Gangopadhyay, M.; Dey, S.; Anoop, A.; Pradeep Singh, N. D. A p-Hydroxyphenacyl-Benzothiazole-Chlorambucil Conjugate as a Real-TimeMonitoring Drug-Delivery System Assisted by Excited-State Intramolecular Proton Transfer. Angew. Chem.Int. Ed., 2016, 55, 4194-4198. (13) (a) Gao, M.; Hu, Q.; Feng, G.; Tang, B. Z.; Liu, B. A fluorescent light-up probe with "AIE + ESIPT" characteristics for specific detection of lysosomal esterase. J. Mater. Chem. B, 2014, 2, 3438-3442. (b) Mei, J.; Leung, N. L. C.; Kwok, R. T. K.; Lam, J. W. Y.; Tang, B. Z. Aggregation-Induced Emission: Together We Shine, United We Soar!. Chem. Rev. 2015, 115, 11718-11940.

(14) Song, H.; Lee, Y. S.; Roh, E. J.; Seo, J. H.; Oh, K.-S.; Lee, B. H.; Han, H.; Shin, K. J. Discovery of potent and selective rhodanine type IKK $\beta$ inhibitors by hit-to-lead strategy. Bioorg. Med. Chem. Lett., 2012, 22, 5668-5674.

(15) Roy, B.; Roy, S.; Kundu, M.; Maji, S.; Pal, B.; Mandal, M.; Singh, N. D. P. Ground-State Proton-Transfer (GSPT)-Assisted Enhanced Two-Photon Uncaging from a Binol-based AIE-Fluorogenic Phototrigger. Org. Lett., 2021, 23, 2308-2313.

(16) Demas, J. N.; Bowman, W. D.; Zalewski, E. F.; Velapoldi, R. A. Determination of the quantum yield of the ferrioxalate actinometer with electrically calibrated radiometers. J. Phys. Chem. 1981, 85, 27662771.

(17) Givens, R. S.; Heger, D.; Hellrung, B.; Kamdzhilov, Y.; Mac, M.; Conrad, P. G.; Cope, E.; Lee, J. I.; Mata-Segreda, J. F.; Schowen, R. L.; Wirz, J. The Photo-Favorskii Reaction of p-Hydroxyphenacyl Compounds Is Initiated by Water-Assisted, Adiabatic Extrusion of a Triplet Biradical. J. Am. Chem. Soc. 2008, 130, 3307-3309.

(18) Sheik-Bahae, M.; Said, A. A.; Wei, T.-H.; Hagan, D. J.; Van Stryland, E. W. Sensitive Measurement of Optical Nonlinearities Using a Single Beam. IEEE J. Quantum Electron. 1990, 26, 760-769.

(19) Ajami, A.; Husinsky, W.; Liska, R.; Pucher, N. Two-photon absorption cross section measurements of various two-photon initiators for ultrashort laser radiation applying the Z-scan technique. J. Opt. Soc. Am. B 2010, 27, 2290-2297.

(20) Jana, A.; Devi, K. S. P.; Maiti, T. K.; Singh, N. D. P. Perylene-3Ylmethanol: Fluorescent Organic Nanoparticles as a Single-Component Photoresponsive Nanocarrier with Real-Time Monitoring of Anticancer Drug Release. J. Am. Chem. Soc., 2012, 134, 7656-7659. 